\title{
Desenvolvimento e validação do ensaio de dissolução para captopril em cápsulas magistrais por CLAE
}

\author{
Roberta de Cássia Pimentel Azevedo, Gislaine Pereira Ribeiro, Magali Benjamin de Araújo* \\ Laboratório Núcleo Controle de Qualidade, Departamento de Farmácia, Universidade Federal de Alfenas
}

*Correspondência:

M. B. Araújo

Departamento de Farmácia

Laboratório Núcleo Controle de

Qualidade

Universidade Federal de Alfenas -

UNIFAL-MG

Rua Gabriel Monteiro da Silva, 714

37130-000 - Alfenas - MG, Brasil

E-mail: magali@unifal-mg.edu.br
O captopril é um fármaco anti-hipertensivo e vasodilatador utilizado na insuficiência cardíaca congestiva e encontra-se disponivel, no mercado brasileiro, sob as formas de comprimidos e cápsulas, sendo estas manipuladas em farmácias. $O$ objetivo deste estudo foi validar o procedimento de dissolução e o método de análise. A avaliação do perfil de dissolução do captopril na forma farmacêutica cápsulas magistrais quando submetidas a diferentes condições de $\mathrm{pH}$, aparatos, velocidades de agitação do meio de dissolução e métodos para quantificação foram alvo deste estudo. As concentrações do fármaco no meio foram determinadas por cromatografia líquida e espectrofotometria. Os resultados mostraram que o método cromatográfico foi o mais adequado para avaliação de captopril na forma farmacêutica cápsulas, enquanto o método espectrofotométrico (recomendado pelas Farmacopéias Brasileira e Americana) apresentou baixa especificidade. $O$ procedimento de dissolução nas condições estabelecidas foi preciso, exato e seletivo. O método foi linear. Com base nos resultados obtidos as condições do teste de dissolução para cápsulas foram o uso de $\mathrm{HCl}$ 0,01 $\mathrm{M}\left(900 \mathrm{~mL}, 37{ }^{\circ} \mathrm{C} \pm 0,5{ }^{\circ} \mathrm{C}\right)$, aparato cesta, $50 \mathrm{rpm}$, tempo de coleta (20 minutos) e quantificação pelo método cromatográfico. Todas as cápsulas apresentaram resultados satisfatórios nos testes de qualidade a que foram submetidas.

\section{INTRODUÇÃO}

As formas farmacêuticas sólidas administradas por via oral são amplamente prescritas na prática médica. A manipulação considerando principalmente a manipulação magistral da forma farmacêutica cápsulas, permite a dosagem do fármaco de forma personalizada, a facilidade de
Unitermos

- Captopril/perfil de dissolução

- Cápsulas/dissolução

- Cromatografia líquida de alta eficiência aquisição do medicamento por um custo inferior ao praticado para o medicamento industrializado e, também, a oportunidade de manipular medicamentos não existentes no mercado farmacêutico.

A absorção do fármaco a partir dessa forma farmacêutica depende da sua liberação, da dissolução ou solubilização sob condições fisiológicas e da sua 
permeabilidade através do trato gastrintestinal (Abdou, 1989; Manadas, Pina, Veiga, 2002; Marcolongo, 2003).

Qualquer fator que altere os processos de desagregação e dissolução da forma farmacêutica poderá afetar diretamente a biodisponibilidade, expressa pela quantidade de fármaco absorvido e velocidade do processo de absorção (Dressman et al., 1988; Storpirtis, Consiglieri, 1995). Desse modo, os testes de dissolução in vitro constituem uma das ferramentas essenciais para avaliação das propriedades biofarmacotécnicas das formulações (Delucia, Sertié, 2004; Peixoto et al., 2005).

Dentre os medicamentos manipulados pelas farmácias, encontra-se o captopril, na forma farmacêutica cápsula, utilizado como anti-hipertensivo e vasodilatador na insuficiência cardíaca congestiva (Korolkovas, 2006; Marcatto et al., 2006).

As Farmacopéias Brasileira $4^{\mathrm{a}}$ edição (F. Bras.) e Americana $30^{\mathrm{a}}$ edição (USP) preconizam a técnica da espectrofotometria na região do ultravioleta para determinação da percentagem de cedência no ensaio de dissolução para captopril na forma farmacêutica comprimidos. Na F. Bras. relata-se o comprimento de onda 212 nm e utilização de ácido clorídrico $0,1 \mathrm{M}$ como meio de dissolução e, por outro lado, a USP preconiza o comprimento de onda $205 \mathrm{~nm}$ e como meio de dissolução ácido clorídrico 0,01 M (Farmacopéia Brasileira, 2002; USP, 2007).

A literatura registra trabalhos publicados envolvendo a avaliação da qualidade de cápsulas manipuladas e comprimidos de captopril (Marcatto et al., 2006; Peixoto, et al., 2005; Valentini, Sommer, Matioli, 2004). Para a forma farmacêutica cápsula contendo captopril não existe, até o momento, monografia oficial, o que vem mostrar a necessidade de desenvolvimento e validação de um ensaio de dissolução que possa prever o comportamento in vitro desse fármaco manipulado com freqüência em farmácias.

Considerando as diferentes condições apresentadas pelos códigos oficiais para o ensaio de dissolução da forma farmacêutica comprimido contendo captopril e, em relação ao processo de manipulação de cápsulas, temos que a tecnologia da fabricação e os diluentes empregados podem afetar a dissolução do fármaco, o presente trabalho tem como objetivo o estudo envolvendo o perfil de dissolução de cápsulas contendo captopril, visando à padronização do teste de dissolução para essa forma farmacêutica e a avaliação da qualidade das cápsulas em relação ao peso médio, desintegração, identificação, limite de dissulfeto de captopril, teor e uniformidade de conteúdo.

\section{MATERIAL E MÉTODOS}

\section{Material}

Foram avaliadas cápsulas de captopril 25 mg manipuladas por três diferentes farmácias, denominadas formulações A, B e C, respectivamente. Como especialidade farmacêutica foi empregada o Capoten ${ }^{\circledR}$ comprimidos $25 \mathrm{mg}$. Utilizou-se captopril, substância química de referência (Pharma Nostra, lote 5103-04201C, validade 06/07), que foi validado a partir de padrão primário (F. Bras. lote 1001, teor 99,61\%) por cromatografia líquida de alta eficiência (CLAE), o qual apresentou teor de 99,13\%. Utilizou-se também dissulfeto de captopril (FAFAR-UFMG, lote 052211, teor $94,00 \%$ ).

Para desenvolvimento e validação do ensaio de dissolução foram utilizados metanol (grau HPLC, Vetec ${ }^{\circledR}$ ), ácido fosfórico $\left(\operatorname{Vetec}^{\mathbb{}}\right)$ e ácido clorídrico $\left(\operatorname{Vetec}^{\circledR}\right)$.

Foram utilizados os seguintes equipamentos: aparelho para ensaio de dissolução (Nova Ética ${ }^{\circledR}$ modelo 299-6), equipado com seis cubas; espectrofotômetro UV/Vis (Shimadzu ${ }^{\circledR}$ modelo UV-1601PC); cromatógrafo (Shimadzu ${ }^{\circledR}$ modelo série $10 \mathrm{VP}$, com sistema de integração CLASS-VP 5.05, bomba LC-ADVP, degaseificador DGU-14 A, injetor manual $7725 \mathrm{i}$ com loop de $20 \mu \mathrm{L}$, detector UV/Vis SPD-10AVP, válvula FCV-10ALVP, forno de coluna CTO-10AVP, controlador SCL-10AVP versão 5.33), bomba de vácuo (Nova Técnica ${ }^{\circledR}$ ), sistema de purificação de água (TKA ${ }^{\circledR}$ modelo LAB- HPW) e medidor de $\mathrm{pH}$ (Marconi ${ }^{\circledR}$ modelo PA 200).

Foram utilizadas coluna C-18, $10 \mathrm{~cm}$ de comprimento, $4,6 \mathrm{~mm}$ de diâmetro interno e $5 \mu \mathrm{m}$ de tamanho de partícula (Supelco ${ }^{\circledR}$ ), pré-coluna C-18 com $2 \mathrm{~cm}$ de comprimento, 4,6 mm de diâmetro interno e $5 \mu \mathrm{m}$ de tamanho de partícula $\left(\right.$ Supelco $\left.{ }^{\circledR}\right)$.

\section{Métodos}

\section{Peso médio}

A determinação de peso foi realizada segundo critérios estabelecidos pela Farmacopéia Brasileira (1988). Vinte cápsulas obtidas das formulações $\mathrm{A}, \mathrm{B}$ e $\mathrm{C}$ respectivamente, foram pesadas individualmente e, após remoção do conteúdo, foram novamente pesadas. Determinou-se o peso do conteúdo de cada cápsula pela diferença de peso entre a cápsula cheia e a vazia. Com os valores obtidos, determinou-se o peso médio do conteúdo e os desvios individuais em relação ao peso médio. A variação individual permitida é de \pm $10 \%$ em relação ao peso médio. Podem ser toleradas não mais que duas unidades fora do limite especificado, em relação ao peso médio do conteúdo, porém, nenhuma poderá estar acima ou abaixo do dobro das percentagens indicadas. 


\section{Desintegração}

O teste de desintegração para as cápsulas das formulações A, B e C, foi realizado segundo critérios estabelecidos pela Farmacopéia Brasileira (1988) Foram utilizadas 6 unidades de cada amostra, nas seguintes condições: meio: água como meio de desintegração, temperatura de $37{ }^{\circ} \mathrm{C}$ $\pm 1{ }^{\circ} \mathrm{C}$ e tempo de desintegração de 45 minutos.

Identificação, teor de captopril e de dissulfeto de captopril e uniformidade de conteúdo

Estas análises foram realizadas em cromatógrafo líquido de alta eficiência com detecção a $220 \mathrm{~nm}$, vazão de $1 \mathrm{~mL} / \mathrm{min}$ e temperatura do forno de $25^{\circ} \mathrm{C}$. Os ensaios foram realizados conforme procedimento farmacopeico para captopril na forma farmacêutica comprimidos (Farmacopéia Brasileira, 2002). A fase móvel (FM) composta de mistura de ácido fosfórico $0,11 \%(\mathrm{v} / \mathrm{v})$ e metanol (45:55) foi filtrada através de membrana de celulose regenerada, com porosidade de $0,45 \mu \mathrm{m}$, sob vácuo. Para o preparo da solução padrão, dissolveu-se, analiticamente, $25 \mathrm{mg}$ de captopril padrão secundário na FM em balão volumétrico de $25 \mathrm{~mL}$ com auxílio de $750 \mu \mathrm{L}$ de solução de dissulfeto de captopril $1000 \mu \mathrm{g} / \mathrm{mL}$ em FM (concentração final captopril padrão secundário $1000 \mu \mathrm{g} / \mathrm{mL}$ e dissulfeto de captopril $30 \mu \mathrm{g} / \mathrm{mL}$ ). Para o preparo das soluções das amostras A, B e C, pesaram-se, analiticamente, a partir do peso médio das cápsulas, o equivalente a $25 \mathrm{mg}$ de captopril, que foram transferidos para balão volumétrico de $25 \mathrm{~mL}$ com auxílio de $15 \mathrm{~mL}$ de FM. Levou-se ao banho de ultra-som por 15 minutos e completou-se o volume com FM. As soluções padrão e amostras foram filtradas e injetadas no cromatógrafo $(20 \mu \mathrm{L})$, em quintuplicata, sendo registrados os cromatogramas e as respectivas áreas dos picos.

Para o teste de uniformidade de conteúdo foram analisadas 10 unidades, individualmente, de cada formulação, as quais foram transferidas para balão volumétrico de 25 $\mathrm{mL}$ e adicionaram cerca de $15 \mathrm{~mL}$ de FM. Levou-se ao banho de ultra-som por 15 minutos e completou-se o volume com o mesmo solvente. As condições cromatográficas foram iguais às empregadas no teor de captopril. Foi realizada uma injeção de cada uma das dez unidades das amostras das formulações A, B e C. A partir das áreas foram determinados os teores de fármaco por unidade testada, expresso em percentagem do valor rotulado e calculado a estimativa do desvio padrão relativo (USP, 2007).

\section{Desenvolvimento do ensaio de dissolução}

Foram testadas diversas condições para o ensaio de dissolução das cápsulas, as quais estão indicadas na Tabela I objetivando-se a definição das condições mais adequadas. Para cada condição realizou-se o perfil de dissolução de cada amostra das formulações A, B e C, retirando-se as alíquotas nos tempos de 5, 10 15, 20 e 45 minutos, e repondo-se o mesmo volume de meio a $37 \pm 0,5^{\circ} \mathrm{C}$. As alíquotas coletadas foram filtradas em papel de filtro quantitativo. As concentrações do fármaco no meio de dissolução foram determinadas empregando-se a cromatografia líquida de alta eficiência (fase móvel constituída de mistura de ácido fosfórico $0,11 \%(\mathrm{v} / \mathrm{v})$ e metanol (45:55), coluna $\mathrm{C} 18$, vazão da fase móvel de $1 \mathrm{~mL} /$ minuto, detecção no ultravioleta a $212 \mathrm{~nm}$ ) e espectrofotometria (meio de dissolução como solvente e detecção no ultravioleta a $212 \mathrm{~nm}$ ).

\section{Validação do ensaio de dissolução e do método de quantificação}

A validação do ensaio de dissolução e do método de quantificação foi embasada em critérios estabelecidos pela Resolução RE no 899, de 29 de maio de 2003 da Agência Nacional de Vigilância Sanitária (ANVISA), Official Methods of Analysis of AOAC International (AOAC INTERNATIONAL, 1995) e International Conference on Harmonization (ICH, 1995), através da determinação dos parâmetros de linearidade, precisão, especificidade/ seletividade, estabilidade e exatidão.

TABELA I - Condições avaliadas no desenvolvimento do ensaio de dissolução para cápsulas de captopril (formulações $A, B$ e C).

\begin{tabular}{lcccc}
\hline Condição & 1 & 2 & 3 & 4 \\
\hline Meio $(900 \mathrm{~mL})$ & ácido clorídrico & ácido clorídrico & ácido clorídrico & ácido clorídrico \\
& $0,1 \mathrm{M}$ & $0,1 \mathrm{M}$ & $0,01 \mathrm{M}$ & $0,01 \mathrm{M}$ \\
Aparato & cesta/pá & cesta/pá & cesta/pá & cesta/pá \\
Velocidade de agitação (rpm) & 50 & 100 & 50 & 100 \\
Método de quantificação & UV/CLAE & UV/CLAE & UV/CLAE & UV/CLAE \\
\hline
\end{tabular}




\section{Linearidade}

Foi estabelecida através da construção da curva de calibração, obtida com soluções de captopril padrão secundário nas concentrações de 15, 19, 23, 27 e $30 \mu \mathrm{g} / \mathrm{mL}$, preparadas em $\mathrm{HCl}$ 0,01 M e avaliada pelo método de CLAE.

\section{Precisão (método de análise)}

Foram feitas seis injeções, no mesmo dia (intra-dia) e seis injeções em um segundo dia (inter-dia), de captopril padrão secundário na concentração de $25 \mu \mathrm{g} / \mathrm{mL}$. As médias das determinações e a estimativa dos DPR foram calculadas.

\section{Precisão (procedimento de dissolução)}

Seis cápsulas das formulações A, B e C, respectivamente, foram submetidas ao ensaio de dissolução no mesmo dia (intra-dia) e em um segundo dia (inter-dia), nas condições padronizadas para o ensaio de dissolução. Foi feita uma injeção de cada amostra. A percentagem dissolvida de captopril foi determinada por CLAE. As médias das determinações e a estimativa dos DPR foram calculadas.

\section{Especificidade (procedimento de dissolução)}

Padrão de captopril (concentração $=8,3 \mu \mathrm{g} / \mathrm{mL}$ ), dissulfeto de captopril (concentração $=0,9 \mu \mathrm{g} / \mathrm{mL}$ ), padrão de captopril adicionado de dissulfeto de captopril (concentração $=8,3+0,9 \mu \mathrm{g} / \mathrm{mL}$ ) e placebos correspondentes ao processo de manipulação das cápsulas das formulações $\mathrm{A}, \mathrm{B}$ e C, foram submetidos às condições padronizadas para o ensaio de dissolução, exceto a velocidade de agitação que foi de $150 \mathrm{rpm}$. Após 1 hora, as amostras foram filtradas e avaliadas pelo método espectrofotométrico e cromatográfico.

\section{Estabilidade (método de análise)}

Foram preparadas soluções de captopril padrão secundário (concentração $=27,7 \mu \mathrm{g} / \mathrm{mL}$ ), e das amostras das formulações $\mathrm{A}, \mathrm{B} \mathrm{e} \mathrm{C}$, as quais foram mantidas em temperatura ambiente $\left(25^{\circ} \mathrm{C} \pm 0,2{ }^{\circ} \mathrm{C}\right)$, por um período de 24 horas. As amostras foram analisadas por CLAE.

\section{Estabilidade (procedimento de dissolução)}

Foram preparadas soluções de captopril padrão secundário (concentração $=27,7 \mu \mathrm{g} / \mathrm{mL}$ ) e das amostras das formulações $\mathrm{A}, \mathrm{B}$ e $\mathrm{C}$, as quais foram submetidas às condições padronizadas para o ensaio de dissolução, mas por um período de 2 horas. As amostras foram analisadas por CLAE.

\section{Exatidão (método de análise)}

Foi avaliada através da adição de quantidades conhecidas de padrão secundário, para obtenção de três níveis de concentração da curva de calibração 15, 23 e $30 \mu \mathrm{g} / \mathrm{mL}$, que foram adicionadas aos placebos das cápsulas das formulações A, B e C, respectivamente. As amostras foram analisadas por CLAE. Foram calculados os valores percentuais correspondentes aos níveis de concentração dos placebos contaminados de cada uma das amostras.

\section{Exatidão (procedimento de dissolução)}

A exatidão foi determinada pelo método do placebo contaminado. Placebos correspondentes ao processo de manipulação das cápsulas das formulações A, B e C, foram submetidos às condições padronizadas para o ensaio de dissolução, onde quantidades crescentes de padrão secundário foram adicionadas às cubas para obtenção de três níveis de concentração da curva de calibração, 15,23 e $30 \mu \mathrm{g} / \mathrm{mL}$. As amostras foram analisadas por CLAE. Foram calculados os valores percentuais correspondentes aos níveis de concentração dos placebos contaminados de cada uma das amostras.

\section{RESULTADOS E DISCUSSÃO}

\section{Peso médio}

Os resultados do peso médio das cápsulas de captopril, correspondentes às formulações $\mathrm{A}, \mathrm{B} \mathrm{e} \mathrm{C}$, respectivamente, estão apresentados na Tabela II. Nenhuma cápsula analisada ficou fora dos limites especificados demonstrando homogeneidade de peso.

\section{Desintegração}

Todas as amostras das farmácias atenderam às especificações estabelecidas pela Farmacopéia Brasileira, ou seja, as cápsulas estavam completamente desintegradas ao final de 45 minutos. A desintegração das cápsulas das formulações A e C ocorreu em 2 minutos e da formulação $\mathrm{B}$ em 3 minutos.

\section{Identificação, teor de captopril e de dissulfeto de captopril}

Os cromatogramas obtidos por CLAE para captopril padrão secundário e cápsulas estão apresentados nas Figuras 1 e 2, respectivamente.

Os cromatogramas do captopril padrão secundário e cápsulas das formulações A, B e C, apresentaram o mesmo 
TABELA II - Valores obtidos na determinação de peso de cápsulas de captopril (formulações A, B e C, respectivamente).

\begin{tabular}{lcccc}
\hline Amostras & Peso médio $(\mathrm{mg})$ & $\mathrm{DPR}(\%)$ & Desvio individual(mg) & $\begin{array}{c}\text { Resultado para o } \\
\text { limite de variação }\end{array}$ \\
\hline formulação A & 117,7 & $1,76(\mathrm{n}=20)$ & 105,9 a 129,5 & De acordo \\
formulação B & 134,0 & $4,74(\mathrm{n}=20)$ & 120,6 a 147,4 & De acordo \\
formulação C & 122,4 & $2,20(\mathrm{n}=20)$ & 110,2 a 134,6 & De acordo \\
\hline
\end{tabular}

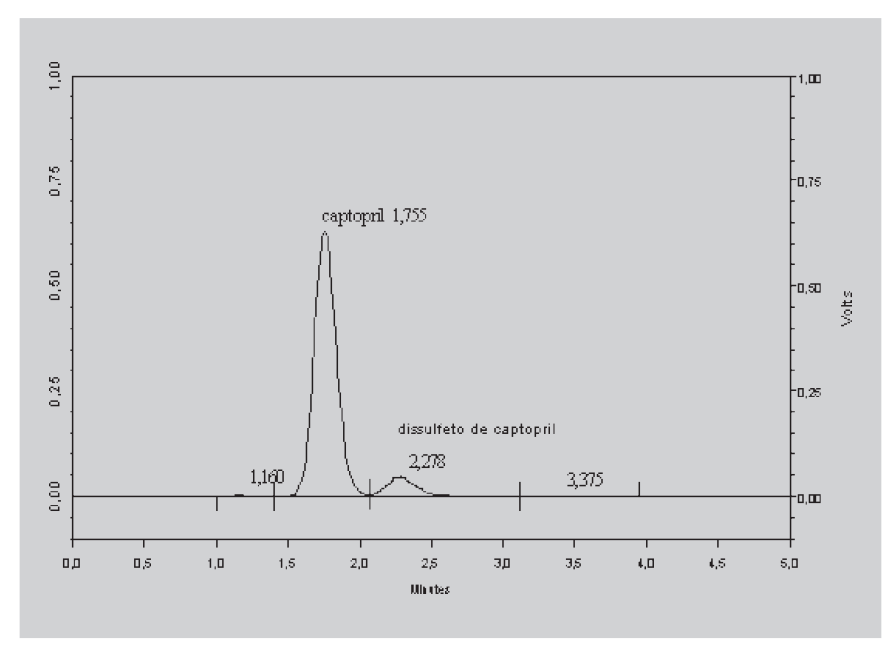

FIGURA 1 - Cromatograma do captopril padrão secundário, fase móvel mistura de ácido fosfórico $0,11 \%$ (v/ v) e metanol (45:55), vazão $1 \mathrm{~mL} / \mathrm{min}$, coluna C-18, comprimento de onda de deteç̧ão $220 \mathrm{~nm}$ e resolução média 2,2 .

perfil e tempos de retenção próximos: 1,755 minutos para o padrão secundário e 1,750 minutos para as cápsulas. Em relação aos picos do dissulfeto de captopril encontrados nas amostras das formulações A, B e C observa-se quantidades inferiores a que foi adicionada no padrão secundário $(30 \mu \mathrm{g} /$ $\mathrm{mL}$ ). Os valores percentuais obtidos referentes ao doseamento, limite de dissulfeto de captopril e uniformidade de conteúdo das formulações A, B e C, estão demonstrados na Tabela III.

Os produtos acabados analisados por CLAE apresentaram valores médios de teores que correspondem a $25,42 \mathrm{mg}, 23,93 \mathrm{mg}$ e 24,68 $\mathrm{mg}$ de captopril para as formulações $\mathrm{A}, \mathrm{B}$ e $\mathrm{C}$, respectivamente, em relação à dosagem

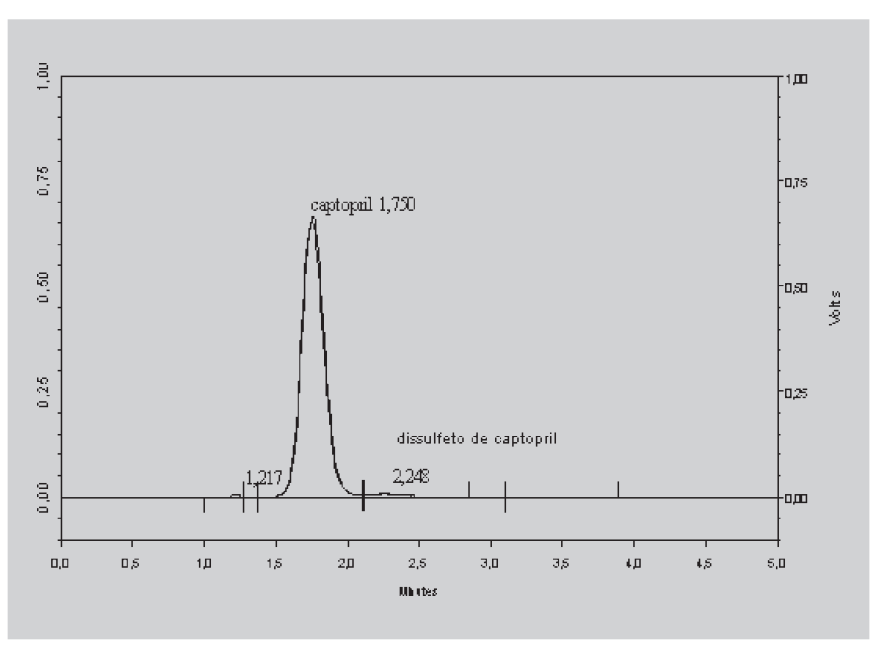

FIGURA 2 - Cromatogramas superpostos do captopril cápsulas das formulações $\mathrm{A}, \mathrm{B}$ e $\mathrm{C}$, fase móvel mistura de ácido fosfórico $0,11 \%$ (v/v) e metanol (45:55), vazão $1 \mathrm{~mL} /$ min, coluna C-18, comprimento de onda de detecção 220 nm e resolução média 2,3 .

declarada, indicando quantidade do fármaco adequada em cada formulação.

O processo de manipulação de cápsulas apresenta menor número de etapas, evitando a exposição excessiva do produto e conseqüente degradação oxidativa do captopril a dissulfeto de captopril. Todas as amostras de cápsulas apresentaram teor de dissulfeto de captopril (Tabela III e Figuras 1 e 2) abaixo de 3,0\%, limite máximo especificado para a forma farmacêutica comprimido (Farmacopéia Brasileira, 2002). Os valores baixos apresentados vêm demonstrar condições de armazenamento adequadas tanto de temperatura como de umidade, favorecendo a estabili-

TABELA III - Valores obtidos na determinação dos teores médios de captopril, de dissulfeto de captopril e da uniformidade de conteúdo (formulações A, B e C).

\begin{tabular}{lccc}
\hline & formulação A & formulação B & formulação C \\
\hline captopril (\%) & 101,70 & 95,70 & 98,70 \\
dissulfeto de captopril (\%) & 0,10 & 1,00 & 1,10 \\
uniformidade de conteúdo (\%) & 97,50 & 98,80 & 101,40 \\
\hline
\end{tabular}


dade dos produtos manipulados.

Os valores de teor de captopril (percentagem do valor rotulado) obtidos no teste de uniformidade de conteúdo, apresentados na Tabela III, correspondem a 24,38 mg, 24,70 mg e $25,35 \mathrm{mg}$ de captopril nas unidades de cápsulas testadas oriundas das formulações A, B e C, respectivamente, demonstrando procedimento de enchimento individual uniforme. As estimativas de DPR inferiores a $6,0 \%$ em cada formulação atendem ao estabelecido pela Farmacopeia Brasileira para a forma farmacêutica cápsula.

\section{Ensaio de dissolução}

Para definir os parâmetros adequados para avaliar a dissolução de captopril na forma farmacêutica cápsulas, diferentes aparatos, meios e velocidades de agitação foram testados (Tabela I). Independentemente da condição empregada, verificou-se que os percentuais de cedência com quantificação espectrofotométrica foram superiores aos da cromatográfica. Com relação ao método de quantificação, a extensa e fácil utilização da espectrofotometria pode justificar o seu emprego, entretanto, este método não permite a determinação do dissulfeto de captopril, por esta razão os percentuais de cedência foram superiores a $100 \%$ e os perfis de dissolução apresentaram-se com maior variabilidade (Tabela IV). O método cromatográfico, além de apresentar execução de técnica relativamente simples e ter o diferencial de ser o método de maior sensibilidade, permite a quantificação do dissulfeto de captopril, indicativo de estabilidade (Valentini, Sommer, Matioli, 2004).

Foi utilizado o delineamento inteiramente casualizado em esquema fatorial $3 \times 2 \times 2 \times 2$ e comparação de médias pelo teste de Tukey ao nível de significância de 5\% para análise estatística dos resultados com quantificação cromatográfica.

Comparando-se as formulações analisadas verificouse que houve diferença estatisticamente significativa $(P<0,05)$ quando se analisava uma ou outra. A formulação $\mathrm{B}$ apresentou maior percentagem de cedência em todas as condições testadas.

Comparando-se os meios utilizados (ácido clorídrico 0,01 M e ácido clorídrico $0,1 \mathrm{M}$ ) verificou-se que houve diferença estatisticamente significativa $(\mathrm{P}<0,05)$ quando se utilizava um ou outro. O meio ácido clorídrico $0,01 \mathrm{M}$ apresentou maior percentagem de cedência do fármaco.

Comparando-se os aparatos utilizados (pá e cesta) verificou-se que houve diferença estatisticamente significativa $(P<0,05)$ quando se utilizava um ou outro. $\mathrm{O}$ aparato cesta apresentou maior percentagem de cedência do fármaco.

Os resultados das análises demonstraram que não houve diferença significativa $(P>0,05)$ em se utilizar à velocidade de agitação de $50 \mathrm{rpm}$ ou de $100 \mathrm{rpm}$. Utilizando velocidade de agitação de $100 \mathrm{rpm}$ a percentagem de cedência alcançou valores em torno de $100 \%$, em apenas 5 minutos de teste, para a formulação A, então foi selecionada a velocidade de agitação de $50 \mathrm{rpm}$ para realização do ensaio de dissolução.

Com base nos resultados obtidos, as condições otimizadas do ensaio de dissolução para captopril na forma farmacêutica cápsulas estão descritas na Tabela V e representadas na Figura 3.

O medicamento referência Capoten ${ }^{\circledR}$ foi submetido às mesmas condições do ensaio de dissolução para forma farmacêutica cápsulas, objetivando-se a reprodução das condições otimizadas. O ensaio de dissolução de captopril na forma farmacêutica cápsulas demonstrou, também, resultados satisfatórios para o medicamento referência, na forma farmacêutica comprimido, como demonstrado na Figura 4.

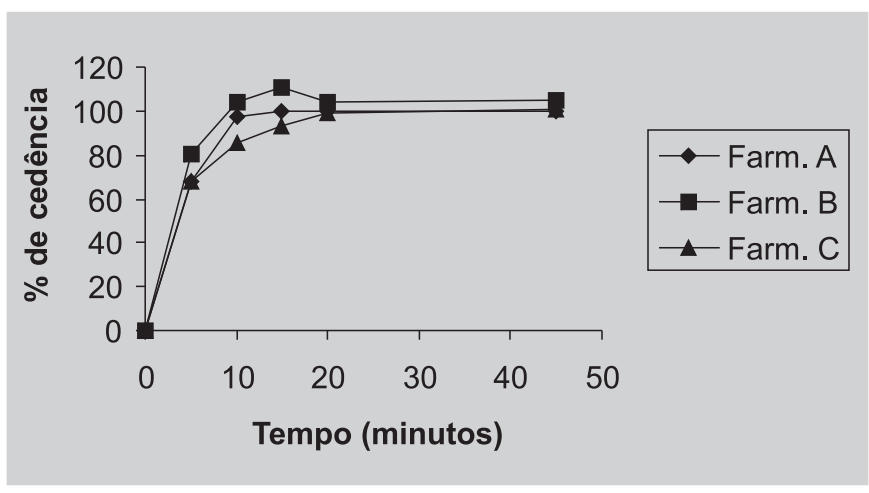

FIGURA 3 - Perfis de dissolução das formulações A, B e $\mathrm{C}$, respectivamente, utilizando ácido clorídrico $0,01 \mathrm{M}$ como meio dissolução $\left(37 \pm 0,5^{\circ} \mathrm{C}\right)$, cesta como aparato, velocidade de agitação de $50 \mathrm{rpm}$ e quantificação cromatográfica em $212 \mathrm{~nm}$.

TABELA IV - Percentagem de cedência das cápsulas de captopril obtidas no tempo de 20 minutos na seguinte condição: ácido clorídrico $0,01 \mathrm{M}$ como meio de dissolução $\left(37 \pm 0,5^{\circ} \mathrm{C}\right)$, cesta como aparato, velocidade de agitação de $50 \mathrm{rpm}$.

\begin{tabular}{lccc}
\hline Quantificação & \multicolumn{2}{c}{$\begin{array}{c}\text { Percentagem de captopril dissolvida no tempo de 20 minutos } \\
\text { formulação B }\end{array}$} & formulação C \\
\hline Espectrofotométrica & 105,31 & 115,21 & 120,32 \\
Cromatográfica & 99,72 & 103,98 & 99,42 \\
\hline
\end{tabular}


TABELA V - Condições otimizadas do ensaio de dissolução para captopril na forma farmacêutica cápsulas.

\begin{tabular}{lc}
\hline \multicolumn{2}{c}{ Parâmetros } \\
\hline meio de dissolução $(900 \mathrm{~mL})$ & ácido clorídrico $0,01 \mathrm{M}$ \\
aparato & cesta \\
velocidade de agitação & $50 \mathrm{rpm}$ \\
método de quantificação & cromatográfico \\
\hline
\end{tabular}

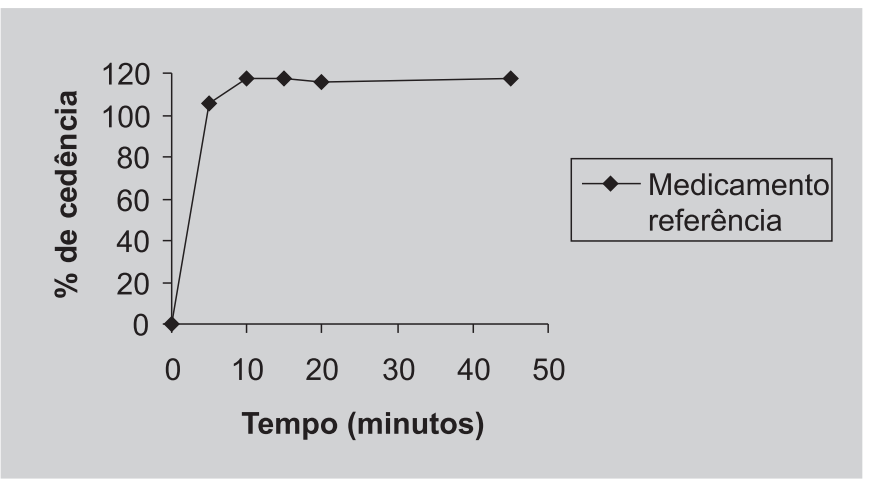

FIGURA 4 - Perfil de dissolução do medicamento referência Capoten ${ }^{\circledR}$, utilizando ácido clorídrico $0,01 \mathrm{M}$ como meio dissolução $\left(37 \pm 0,5^{\circ} \mathrm{C}\right)$, cesta como aparato, velocidade de agitação de 50 rpm e quantificação cromatográfica em $212 \mathrm{~nm}$.

\section{Validação}

As Figuras 5 e 6 apresentam os cromatogramas de captopril padrão secundário e na forma farmacêutica cápsulas, respectivamente. O pico referente ao captopril possui tempo de retenção de, aproximadamente, 1,760 minutos.
A curva de calibração para captopril padrão secundário mostrou linearidade adequada na faixa de 15 a $30 \mu \mathrm{g} /$ $\mathrm{mL}, \operatorname{com~} \mathrm{r}=0,9996$ (coeficiente de correlação). A equação da reta obtida foi: $y=18543 x+91867$. Os valores de DPR inferiores a 5\% (BRASIL, RESOLUÇÃO RE n 899 , 2003 ) intra-dia e inter-dia, considerando o procedimento de dissolução e o método de análise, indicam boa precisão. O espectro de absorção do produto de degradação, dissulfeto de captopril, indicou interferência significativa no comprimento de onda de máxima absorção do captopril inviabilizando o uso do método por espectrofotometria no ultravioleta (Figura 7). Essa interferência não ocorreu para o método cromatográfico, o qual se mostrou adequado para avaliar a dissolução do captopril em cápsulas (Figura 6). A especificidade verificada com o método cromatográfico demonstrou não haver interferência dos excipientes. O fármaco foi estável nas condições avaliadas considerando a temperatura ambiente $\left(25 \pm 0,2^{\circ} \mathrm{C}\right) / 24$ horas apresentando valores de $101,6 \%$ e a $37 \pm 0,5^{\circ} \mathrm{C} / 2$ horas com valores de $99,2 \%$, atendendo a faixa de 98 a $102 \%$ (Marques, Brown, 2002). Na avaliação da exatidão, valores de 96,52 a $102,40 \%$ para o método de análise e de 97,74 a 100,53\% para o procedimento de dissolução, indicam exatidão adequada. Valores entre 95 a 105\% são satisfatórios (Marques, Brown, 2002).

\section{CONCLUSÕES}

Os testes de qualidade para as cápsulas das formulações $\mathrm{A}, \mathrm{B}$ e $\mathrm{C}$ foram considerados satisfatórios em relação ao peso médio, desintegração, identificação, teor de captopril, de dissulfeto de captopril e uniformidade de conteúdo.

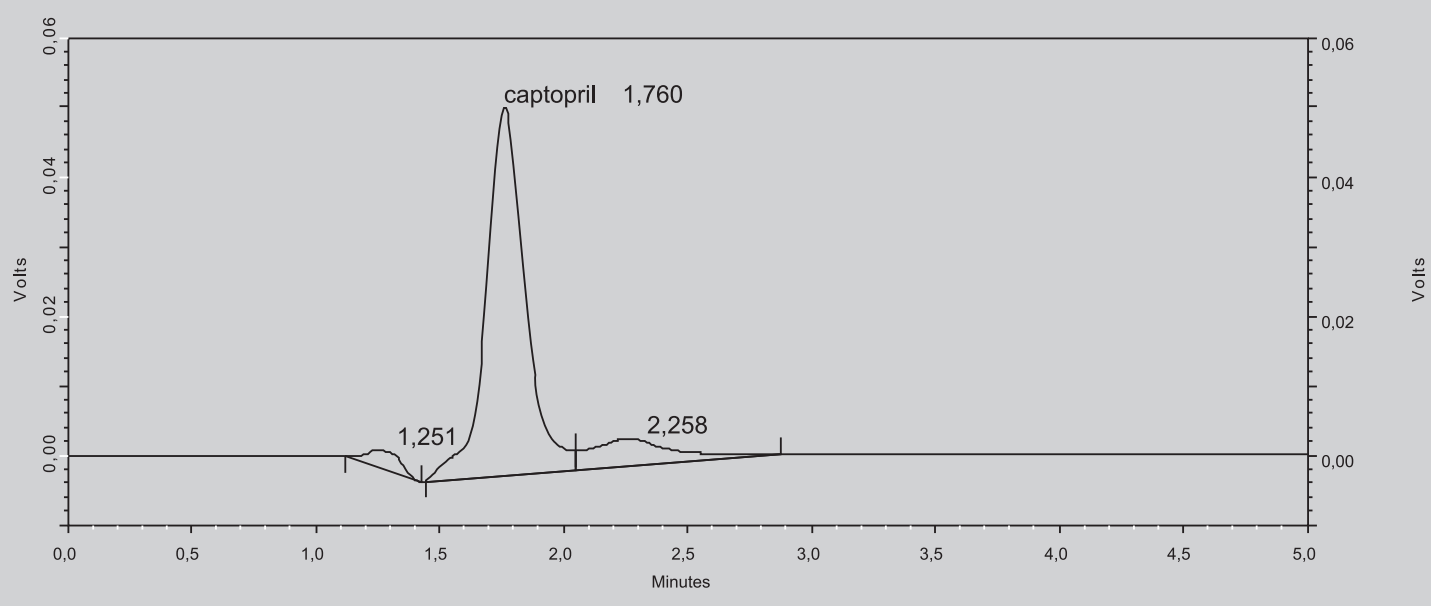

FIGURA 5 - Cromatograma do captopril padrão secundário a $27,7 \mu \mathrm{g} / \mathrm{mL}$ obtido no ensaio de dissolução utilizando cesta como aparato, meio $\mathrm{HCl} 0,01 M\left(37 \pm 0,5^{\circ} \mathrm{C}\right)$ e velocidade de agitação de $50 \mathrm{rpm}$. Condições cromatográficas: fase móvel mistura de ácido fosfórico $0,11 \%$ (v/v) e metanol (45:55), vazão $1 \mathrm{~mL} / \mathrm{min}$, coluna C-18, comprimento de onda de detecção $212 \mathrm{~nm}$. 


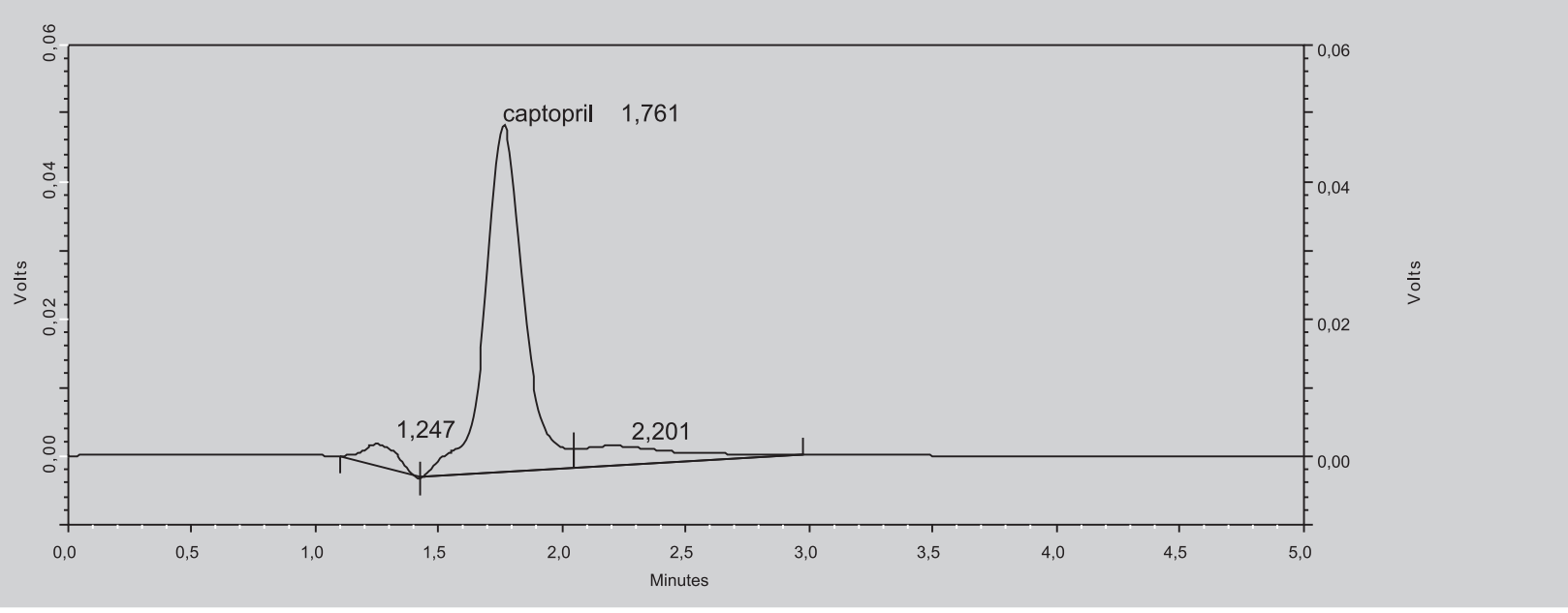

FIGURA 6 - Cromatograma do captopril cápsula a $27,7 \mu \mathrm{g} / \mathrm{mL}$ obtido no ensaio de dissolução utilizando cesta como aparato, meio $\mathrm{HCl} 0,01 M\left(37 \pm 0,5^{\circ} \mathrm{C}\right)$ e velocidade de agitação de $50 \mathrm{rpm}$. Condições cromatográficas: fase móvel mistura de ácido fosfórico $0,11 \%$ (v/v) e metanol (45:55), vazão $1 \mathrm{~mL} / \mathrm{min}$, coluna C-18, comprimento de onda de detecção $212 \mathrm{~nm}$.

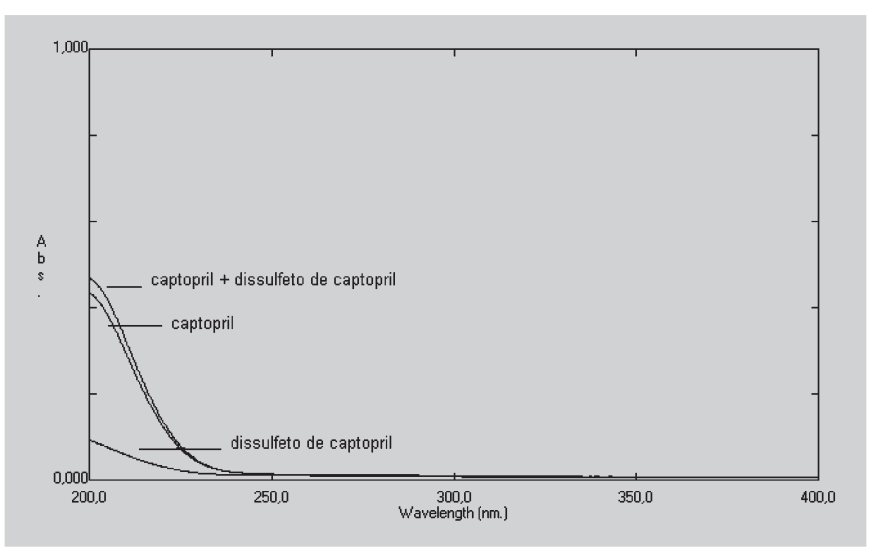

FIGURA 7 - Espectro de absorção na região do ultravioleta do dissulfeto de captopril $(0,9 \mu \mathrm{g} / \mathrm{mL})$, do dissulfeto de captopril adicionado de captopril padrão secundário $(0,9$ $\mu \mathrm{g} / \mathrm{mL}$ e $8,3 \mu \mathrm{g} / \mathrm{mL}$, respectivamente), e do captopril padrão secundário $(8,3 \mu \mathrm{g} / \mathrm{mL})$, após dissolução em ácido clorídrico $0,01 \mathrm{M}$, com aparato cesta a $150 \mathrm{rpm}$ e 1 hora.

No desenvolvimento do ensaio de dissolução, diversas condições foram testadas e os seguintes parâmetros foram otimizados e considerados adequados: ácido clorídrico $0,01 \mathrm{M}\left(900 \mathrm{~mL}\right.$, a $\left.37 \pm 0,5^{\circ} \mathrm{C}\right)$ como meio de dissolução, aparato cesta, velocidade de agitação de $50 \mathrm{rpm}$ e tempo de coleta em 20 minutos demonstrando melhor capacidade de liberação do fármaco.

Em relação à especificidade, o método que melhor expressou tal atributo foi o cromatográfico, pois se conseguiu identificar e quantificar o produto de degradação, dissulfeto de captopril, presente nas cápsulas de captopril, enquanto que o método espectrofotométrico demonstrou valores que satisfazem os critérios de aceitação, porém, com maior variabilidade e menor sensibilidade.

O método proposto para o ensaio de dissolução de cápsulas de captopril por CLAE foi validado, mostrando especificidade, linearidade, precisão, exatidão e estabilidade.

A condição otimizada para o ensaio de dissolução de captopril na forma farmacêutica cápsulas demonstrou também resultados satisfatórios para o medicamento referência, comercializado na forma farmacêutica comprimidos.

\section{ABSTRACT}

\section{Development and validation of dissolution test for captopril in capsules by HPLC}

Captopril is an anti-hypertensive and vasodilator agent utilized in the congestive cardiac insufficiency and it can be commercially found in Brazil in the form of tablets and compounded capsules. The aim of this study was to evaluate the dissolution profile of captopril in capsules dosage obtained from compounded pharmacies, when submitted to different conditions of $\mathrm{pH}$, apparatus, stirring speed of dissolution medium and analytical method, as well the validation of the dissolution procedure and of the method of analysis. The drug concentrations in dissolution medium were determined by liquid chromatography and ultraviolet spectrophotometric. The results showed that the chromatographic method was the most suitable for captopril capsules evaluation, while the spectrophometric method (recommended by the Brazilian and the United States Pharmacopeias) presented low specificity. The dissolution procedure in the selected 
conditions was precise, accurate and specific. The method was linear. With base in the results obtained the conditions of dissolution test for capsules were the use of $0.01 \mathrm{M} \mathrm{HCl}$ $\left(900 \mathrm{~mL}, 37 \pm 0.5^{\circ} \mathrm{C}\right)$, basket apparatus, stirring speed 50 rpm, 20 minutes time and quantification by chromatographic method. All the capsules presented satisfactory results in all the tests assessed.

UNITERMS: Captopril/dissolution profile. Capsules/ dissolution. High performance liquid chromatography.

\section{AGRADECIMENTOS}

Os autores agradecem as farmácias com manipulação, conveniadas ao laboratório Núcleo Controle de Qualidade da UNIFAL-MG, que forneceram as formulações A, $\mathrm{B}$ e C.

\section{REFERÊNCIAS BIBLIOGRÁFICAS}

ABDOU, H.M. Dissolución, biovailability and bioequivalence. Easton: Mack Printing, 1989. 554p.

ASSOCIATION OF OFFICIAL ANALYTICAL CHEMISTS. Official methods of analysis of AOAC. 16.ed. v.1, Arlington, 1995. pt.1, p.18.

BRASIL. Resolução n.899, de 29 de maio de 2003. Adiretoria colegiada da Agência Nacional de Vigilância Sanitária aprova o Guia para Validação de Métodos Analíticos e Bioanalíticos. Diário Oficial da União, Brasília, Seção 1, 2 jun 2003. p.47.

DRESSMAN, J.B; AMIDON, G.L.; REPPAS, C.; SHAH, V.P. Dissolution testing as prognostic tool for oral drug absorption: immediate release dosage forms. Pharm. Rev., v.15, n.1, p.11-22, 1998.

DELUCIA, R.; SERTIÉ, J.A.A. Absorção, biodisponibilidade e bioequivalência de fármacos. In: VALLE, L.B.D.S.; OLIVEIRA-FILHO, R.M.; DELUCIA, R.; OGA, S. Farmacologia integrada. 2.ed. Rio de Janeiro: Revinter, 2004. cap.5, p.35-43.

FARMACOPÉIA Brasileira, 4.ed. São Paulo: Atheneu, 1988. pt.1, p.v.1.

FARMACOPÉIA Brasileira, 4.ed. São Paulo: Atheneu, 2002. pt.2, p.181-181.1.
ICH. International Conference on Harmonization. Validation of analytical procedures: methodology, Q2B (CPMP/ ICH/281/95, 1995. Disponível em: <http://www.fda.gov/ cder>. Acesso em: 15 jun. 2007.

KOROLKOVAS, A. Dicionário terapêutico Guanabara. 2006/2007.ed. Rio de Janeiro: Guanabara Koogan, 2006. p.8.23-8.26.

MANADAS, R.; PINA, M.E.; VEIGA, F. A dissolução in vitro na previsão da absorção oral de fármacos em formas farmacêuticas de liberação modificada. Rev. Bras. Cienc. Farm., v.38, n.4, p.375-399, 2002.

MARCATTO, A.C.; LAMIN, R.; BLOCK, L.C.; BRESOLIN, T.M.B. Análise de cápsulas de captopril manipuladas em farmácias. Rev. Ciênc. Farm. Básica Apl., v.26, n.3, p.221-225, 2006.

MARCOLONGO, R. Dissolução de medicamentos: fundamentos, aplicações, aspectos regulatórios e perspectivas na área farmacêutica. São Paulo, 2003. 117p. [Dissertação de Mestrado. Faculdade de Ciências Farmacêuticas. Universidade de São Paulo].

MARQUES, M.R.C.; BROWN, W. Desenvolvimento e validação de métodos de dissolução para formas farmacêuticas sólidas orais. Rev. Anal., n.1, p.48-51, 2002.

PEIXOTO, M.M.; JÚNIOR, A.F.S; SANTOS, C.A.A; JÚNIOR, E.C. Avaliação da qualidade de comprimidos de captopril dispensados em Feira de Santana-BA. Pharm. Bras., n.47, p.69-73, 2005.

STORPIRTIS, S.; CONSIGLIERI, V.O. Biodisponibilidade e bioequivalência de medicamentos: aspectos fundamentais para o planejamento e execução de estudos. Rev. Farm. Bioquím. Univ. S. Paulo, v.31, p.63-70, 1995.

UNITED States Pharmacopeia. 30.ed. Rockville: United States Pharmacopeial Convention, 2007. p.1611-1612.

VALENTINI, S.R.; SOMMER, W.A.; MATIOLI, G. Validação de métodos analíticos na quantificação de comprimidos de captopril - comparação de metodologias para um programa de garantia de qualidade. Acta Sci. Health Sci., v.26, n.2, p.357-364, 2004.

Recebido para publicação em 13 de junho de 2007 Aceito para publicação em 31 de janeiro de 2008 